\title{
CREATION OF A PUBLICLY ACCESSIBLE RESOURCE FOR INCREASING THE VOLUME OF FREELY DISTRIBUTED MEDICAL DATASETS
}

\author{
Artyom Lobantsev, Artyom Beresnev, Mark Tkachenko, Olga Alekseeva, Natalia Gusarova, \\ Aleksandra Vatian and Anatoly Shalyto \\ ITMO University, Sankt-Petersburg 197101, Russia
}

\begin{abstract}
The lack of data of the required volume and quality is a bottleneck for the widespread use of artificial intelligence technologies in all spheres of human being. This issue is especially acute in medicine. The article discusses the social, motivational and organizational aspects of this problem. As a means to increase the volume of freely distributed medical datasets the creation of a publicly accessible Internet-resource is proposed, which would combine the work of students and young professionals, led by expert radiologists, as well as IT professionals involved in improving markup tools and building datasets. Participating in such kind of work will leverage the motivation of mature specialists and the professional level of young ones. Patients and their attending physicians who wish to promptly receive a second (expert) report on the diagnosis and course of the disease and who agree to use their information anonymously to form datasets will provide the flow of input information. Through an expert survey, we identified the requirements for such a resource. The main business process of such a resource and its system architecture are represented. We conducted experimental studies of the main functionalities of the developed service in terms of meeting the user's requirements as well as a security audit. Quite high estimates were obtained, which allows us to proceed to launch the resource on the platform of the ITMO University (St. Petersburg, Russia).
\end{abstract}

\section{KEYWORDS}

Medical Dataset, Publically Accessible Resource, Radiologist's Motivation

\section{INTRODUCTION}

The principles of machine learning and, more broadly, artificial intelligence are the basis of most modern high-tech solutions in all spheres of human being. This issue is especially acute in medicine, including such areas as medical diagnostics, data analysis, P4-medicine, drug design, etc. However, the effectiveness of systems based on the principles of machine training is critically dependent on sufficient quantity and quality of data. The problem of lack of data, which is especially acute in the field of medical information systems, is largely due to objective reasons: as a rule, the available data cannot cover the whole variety of clinical manifestations of the studied disease, and even the available data for the most part remain unused, since their markup is very resource-intensive procedure.

For example, for diseases associated with the occurrence of neoplasms, the most valuable medical data are extracted from high-tech medical images, primarily CT and MRI images. However, the nomenclature of such diseases is very wide, and, accordingly, clinical cases for each of them are not enough to form a complete dataset for training the neural network. In addition, manual segmentation is extremely slow and tedious, and an expert physician can make mistakes due to fatigue. Noise, parameter variations, and artifacts of the equipment on which the image is formed introduce additional difficulties in this process.

To solve the indicated problem, different approaches are proposed, including:

the use of neural networks such as U-Net [Ronneberger], focused on processing small datasets;

problem-oriented data augmentation;

- creation of synthetic datasets, for example, parametric modeling of high-tech medical images through generative adversarial networks (GAN) [Han, Jin]; 
- the simultaneous use of multimodal medical data having a different nature and presentation format, but complementing each other in essence, like MRI-image and radiologist report compiled from it [Lahat, Rajalingam].

However, all of the above approaches remain alleviative to some extent, since they cannot completely replace datasets collected in real clinical conditions and marked up by professional radiologists. The lack of data of the required volume and quality is a bottleneck for the widespread use of artificial intelligence technologies. It must be emphasized that this problem is not of narrow medical, but of a complex nature and affects such aspects of ICT and modern society interactions as medicine, especially in the diagnosis of neoplasms. The open datasets such as BraTS, LIDC-IDRI, CheXpert, etc. are in great shortage.

In addition, in most cases, open datasets cannot be a panacea. They are created mainly for academic purposes - for example, to assess the quality of models, for research, hypothesis testing, but not for final development. In general, it rarely happens that a dataset not assembled for a specific task is completely representative.

Thus, the creation of means for the formation of freely distributed medical datasets of proper volume and quality is an urgent problem. It must be emphasized that this problem is not of narrow medical, but of a complex nature and affects many aspects of ICT and modern society interactions. From the point of view of radiologists, these are motivation, skills and competencies as well as quality of working life. From the point of view of patients, these are trust, security and privacy of data provided. In terms of their interaction, that is creating of virtual community. Finally, from the point of view of society as a whole, it is improving of health and quality of life.

The article discusses the possibility of creating an Internet resource that integrates all of the above aspects, which we will consider on the example of an important problem - forming freely distributed datasets of high-tech medical images supporting ICT-diagnostics of different types of lung and brain neoplasms. Technological, organizational and educational aspects of such a resource, as well as data privacy issues are considered.

\section{BACKGROUND AND RELATED WORKS}

According to [Kumar, Norouzi], the markup of the medical image for the dataset comprises identifying region of interest, in particular, the separation of healthy and pathological areas (locating tumor, lesion and other abnormalities). In addition, it is highly desirable to accompany each image with a detailed textual radiologist's report.

When creating a means to support the markup of high-tech medical images a number of interrelated questions arise: who performs the markup, what tools are used for it, where do source data come from. Substantially, these questions correspond to three groups of requirements, which can be identified as follows: community aspect (physical and virtual) - C; labour market aspect (outsourcing, integration, mobility) - L; security aspect (trust - security - privacy) - S. Note that the brief notation will be used in table 1.

Regarding the first question, a shortage of qualified radiologists has become a serious worldwide problem nowadays [Allyn, Clinical, Orenstein]. For example, according to the report [Clinical], hospitals in United Kingdom are short of at least 1,104 radiologist to keep up with the demand for diagnostic and interventional radiological care. In developing countries, the situation is even worse [Fornell]. According to the World Health Organization, over half the people in the world are without access to diagnostic and interventional radiology services [Improving]. In these conditions, large hospitals widely use outsourcing, overtime and locum cover, primarily for urgent and interventional radiology, thereby further reducing the circle of specialists who are ready to engage in marking up datasets.

The answer to these challenges is commercial teleradiology, which allows the patient to get an opinion on his medical image from a remote radiologist [Orenstein]. For example, in China teleradiology helps alleviate some of the drawbacks associated with practicing in rural areas [Allyn]. However, as practice shows, teleradiology has so far been developed only in the most advanced countries. In Russia, for example, there are no such services.

In the literature, the problems of motivation of young radiologists, especially students and clinical residents, are widely studied [Goldman, Taylor, Yen]. The work [Taylor] shows declining medical student interest in radiology. As demotivating factors, students note the lack of practice, the inability to get enough 
experience with real medical images, the lack of contact with practicing radiologists. Among the motivating factors, young radiologists, along with the level of salary, note influence of a mentor, perceived job satisfaction, intellectual stimulation and competitiveness of the process [Goldman], as well as competitiveness and professional growth [Yen]. However, the existing worldwide training schemes for young radiologists use these factors very little (see, for example, [Pool]).

As mentioned in [Sunshine], increased productivity is the predominant way to ease the radiologist shortage. Therefore, the organization of the workspace and tools is especially important. The classic workplace of the radiologist is a stationary hardware-software complex, aggregating visualization tools for every kind of medical images, as well as means of communication, storage, etc. [Rugerio]. For marking a specific type of medical image (only CT and MRI images), this equipment is clearly redundant, moreover, it deprives the radiologist of the necessary mobility. That's why, in recent years, more and more portable solutions for the operational support of the work of a radiologist appear in clinical practice (see, for example, [Horos, Mechtash]). A detailed review of such solutions can be found in the work of the authors of present article [Boitsov]. Their development follows the path of maximally simplifying the work of radiologists while maintaining their personal context and marking accuracy; for this, machine-learning technologies are widely used. For example, the toolkit developed by the authors of present article [Boitsov] provides the radiologist with the opportunity of his choice to work in an interactive (semi-automated) or non-interactive (automated) mode. This achieves marking accuracy, measured in Dice coefficient, up to 0.9104 and 0.8333 respectively, along with a high working speed (approx. $15 \mathrm{~s}$ in interactive mode and $5.8 \mathrm{~ms}$ in non-interactive mode).

The last but not least point is where source data come from. The most interesting for the formation of the dataset are real and operatively obtained images of patients with specific diseases, accompanied by a detailed description of the course of the disease, which would allow the radiologist to draw the most accurate report. However, such information falls under a number of laws on the protection of personal data, medical information, etc. For example, the main document concerning the basics of protecting the health of citizens in the Russian Federation is [Federal]. In accordance with this law, the patient's data can be used for telemedicine purposes, for making a decision on the need for face-to-face admission or for treatment correction, but subject to a previously defined diagnosis and a course of treatment prescribed at the face-to-face appointment. Thus, the patient or his attending physician has the right to use the services of teleradiology to obtain a second (expert) opinion on the disease, and ensuring the security of the data when sending it becomes the main problem.

As world practice shows, a promising way to solve this problem in medicine is to use blockchain technology [Vazirany]. Blockchain allows you to create a distributed secure registry of medical records. Given the delicacy of information, access to records can be limited: if the patient and his attending physician agree to show the information to another specialist, they will digitally sign and the system will open access to the specified person.

Combining all of the above, we can conclude that an effective way for increasing the volume of freely distributed medical datasets can be the creation of a publicly accessible Internet resource that works on the principle of teleradiology. The resource will combine the work of students and young professionals, led by expert radiologists, as well as IT professionals involved in improving markup tools and building datasets. Participating in such kind of work will leverage the motivation of mature specialists and the professional level of young ones. Patients and their attending physicians who wish to promptly receive a second (expert) report on the diagnosis and course of the disease and who agree to use their information anonymously to form datasets will provide the flow of input information. The commercialization of such a resource should become a separately discussed topic, but it is clear that by attracting students and young specialists, the price of services for patients will be significantly lower than when contacting a large teleradiological company.

As far as the authors know, resources of this type in developing countries, in particular, in Russia, are not yet available, and this gap should be filled.

Thus, the authors of this article set themselves the following tasks:

1. to identify the applications requirements for Internet resource of such kind;

2. to develop the main business process of such a resource and its system architecture;

3. to conduct experimental studies of the main functionalities of the developed service in terms of meeting the user's requirements. 


\section{REVEALING OF RESOURCE REQUIREMENTS}

To create a list of requirements for the resource under consideration, we conducted an analysis of development practices of medicine resources of analogous types. Besides, we interviewed expert radiologists, students and clinical residents, each of whom independently described his own vision of business process supported by the resourse under consideration as well as it's desirable usability characteristics. The total number of respondents was 15 people, the average work experience of 6.3 years with a range from 0 to 21 years.

Identified requirements are presented in table. 1. In addition, it also shows what enlarged aspects of creating a resource (see section 2) each of them corresponds to.

Table 1. Resourse requirements

\begin{tabular}{|c|c|}
\hline Aspect & Reguirement \\
\hline $\mathrm{S}$ & 1. Compliance with legislation on the protection of personal data. \\
\hline $\mathrm{S}$ & $\begin{array}{l}\text { 2. Unhindered access of the patient to his own medical data to eliminate the obstacle for patients to receive } \\
\text { copies of their medical records or transfer them to another health care provider. }\end{array}$ \\
\hline S & $\begin{array}{l}\text { 3. The integrity and lack of bias of medical data. At any stage, the system must ensure the integrity of the } \\
\text { data, versioning and unambiguous authorship of the data itself and its changes. At the same time, it is } \\
\text { necessary to ensure that the medical data has not been discreetly changed by anyone, including doctors and } \\
\text { the patients themselves. }\end{array}$ \\
\hline $\mathrm{L}, \mathrm{S}$ & $\begin{array}{l}\text { 4. For the system owner - receiving a marked-up quality dataset, with multi-parameter additional attribute } \\
\text { attribution. }\end{array}$ \\
\hline $\mathrm{C}, \mathrm{L}$ & $\begin{array}{l}\text { 5. Minimum requirements for the user to start using the resource (without installing special client software } \\
\text { and with the possibility of using common, including mobile, platforms). }\end{array}$ \\
\hline $\mathrm{C}, \mathrm{L}$ & $\begin{array}{l}\text { 6. Lack of strict regulation on the format of the input data (the patient can present data in a variety of } \\
\text { graphic formats). }\end{array}$ \\
\hline $\mathrm{C}$ & $\begin{array}{l}\text { 7. Lack of requirements for the level of medical knowledge for patient users (maximum use of data entry } \\
\text { wizards, refusal of medical terminology in patient interfaces, nevertheless maintaining its availability in } \\
\text { the form of hints). }\end{array}$ \\
\hline $\mathrm{C}, \mathrm{L}, \mathrm{S}$ & $\begin{array}{l}\text { 8. For rediologists-experts - ease of use of the service, the ability to develop their professional level and } \\
\text { professional impact. }\end{array}$ \\
\hline $\mathrm{C}, \mathrm{L}$ & 9. Support for a professional community, maintaining a rating of experts on consensus assessment. \\
\hline $\mathrm{C}, \mathrm{L}$ & $\begin{array}{l}\text { 10. For students and clinical residents - easy access to real anonymous data and the result of evaluations of } \\
\text { practicing experts. }\end{array}$ \\
\hline
\end{tabular}

\section{BUSINESS PROCESS AND SYSTEM ARCHITECTURE}

An analysis of the requirements presented in Table 1 shows that the most suitable architecture option for the developed resource is the implementation of a modular Web service, with data storage in the form of a private block chain.

Figure 1 shows a use case diagram describing the capabilities of a resource. The main actors include: the patient, an expert doctor, trainee (medical student or clinical resident) and the owner of the portal. Consider the goals of using the portal and the key cases for these actors.

Patient. The patient's goal is to obtain a qualified, accessible consultation in the form of decoding of an MRI or CT scan from several specialists. Before starting work, the Patient performs registration, places his pictures in an accessible format, and fills out a medical history, thereby forming a request. The patient's personal data is entered into the system anonymously, that is, the full name, exact date and place of birth, as well as document numbers, are not stored. We note, however, that the architecture of the system will allow a transparent transition, if necessary, to the storage of such data. After processing the images by expert doctors, the Patient can get access to all reports on his images from all the experts who processed the images, and the rating of experts and their portfolio is available to the patient.

Expert doctor. The purpose of using the portal for an expert doctor is to create a personal portfolio, expand medical practice, present your skills and level of expertise, indirectly evaluate your skills and level of expertise, discuss complex cases in the professional community, train medical students and clinical residents. 
Before starting work, an expert doctor can register in the system, fill out an expert questionnaire, optionally indicating his name, academic degree, publication list, his contacts, etc. After that, the expert will have access to a list of patient applications with selection by fields of history, area of images, etc. An expert doctor, working in the system, carries out the markup of images, gives his report. For each report, assessments of other experts on this application and indicators of the degree of consensus of experts are available to him. The doctor can repeat the analysis of the application by creating its new edition. Relevant and accessible to other experts is the latest version of report. In addition, an expert doctor can create applications for the analysis of images in order to obtain the opinions of other experts. If the expert doctor is a mentor, then he can form sets of applications for his students to implement various pedagogical tasks.

Trainee. Actor trainee is a medicine student or intern (clinical resident). His purpose is the acquisition or development of competencies in the field of radiology. This actor has access to registration, assignment to a mentor (with his consent), study of reports for familiarization with the selection according to the fields of anamnesis and training sessions when Trainee receives an application, either independently or from a mentor, and performs its analysis with automatic comparison of results with expert estimates through consensus evaluation algorithms.

Portal owner. The owner of the portal, in addition to the humanistic goal of expanding access to qualified medical care, aims to create a high-quality dataset with marked MRI and CT images. The value and difference of this dataset is in additional attribution, namely in the presence of a connection with the anamnesis, the availability of the author rating report, the availability of data on the author report and data on the consensus assessment of experts. The generated dataset can be used for scientific research, as well as to improve the quality of existing diagnostic tools based on machine learning.

Separately, we note that gaining full access to the dataset is possible only for this role. No roles are available to delete report. Also outside the scope of the article are issues of managing electronic digital certificates. In the system, they are solved by using an external PKI.

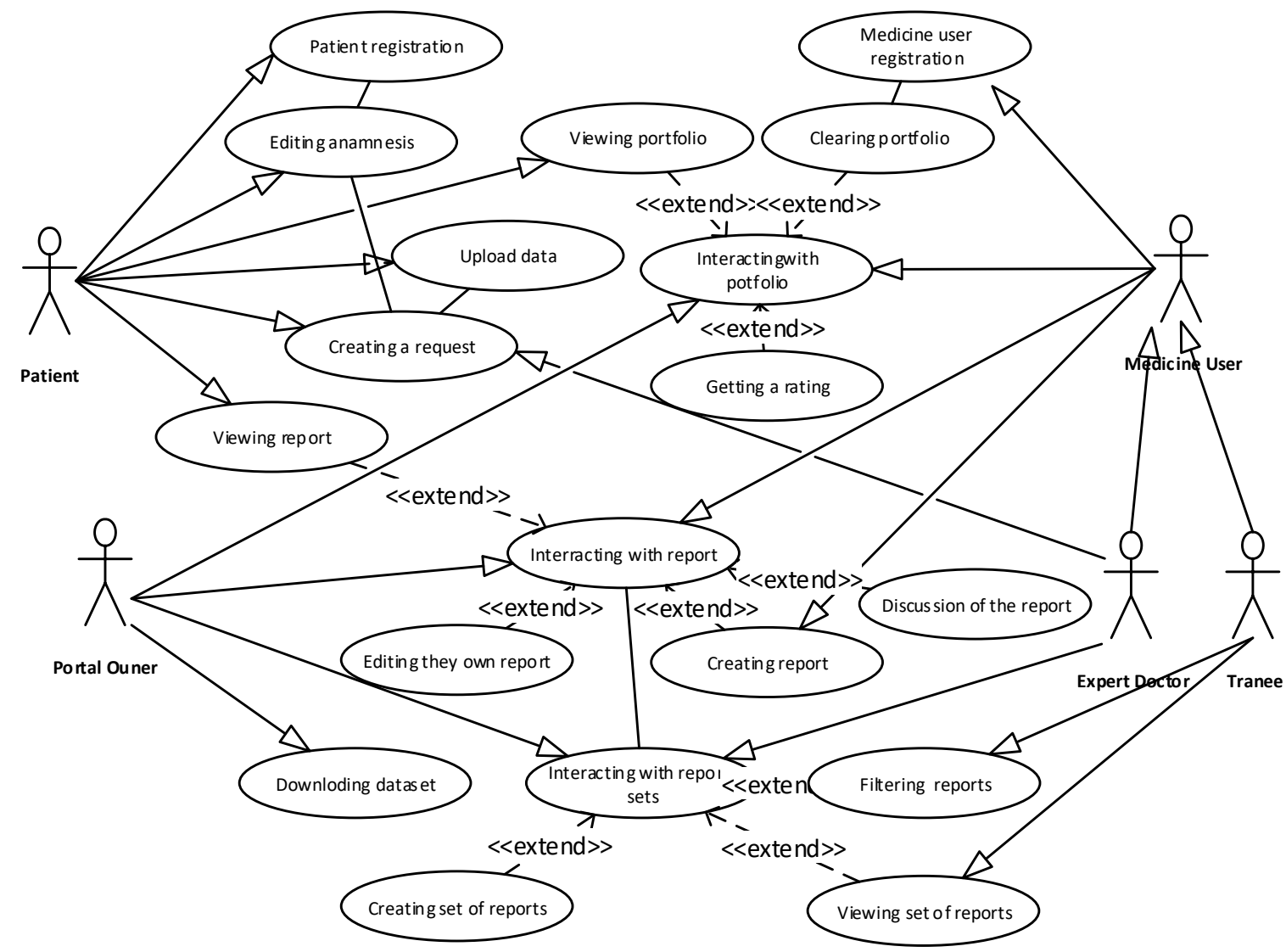

Figure 1. Use Case diagram 
The system architecture of the resource is shown in Figure 2. The client application runs in the browser. The server part of the resource has a modular structure. The Information Resource Interaction Service, deployed on a separate platform, interacts directly with the client. This is a key component of the system through which all the other components, as well as the external PKI service, work. The Information Resource Interaction Service component performs authentication, authorization, interaction with the user interface, as well as the processing of all data coming from other components of the system. The DataConvertation module is functioning on the same physical node, which is designed to automatically bring the data downloaded by the patient to prepare images for marking by an expert. Currently, most raster formats, PDF, DOC $\backslash$ DOCX, RTF and the specialized DICOM format are accepted. The remaining components of the system are hosted on a virtualization platform on separate Linux virtual machines.

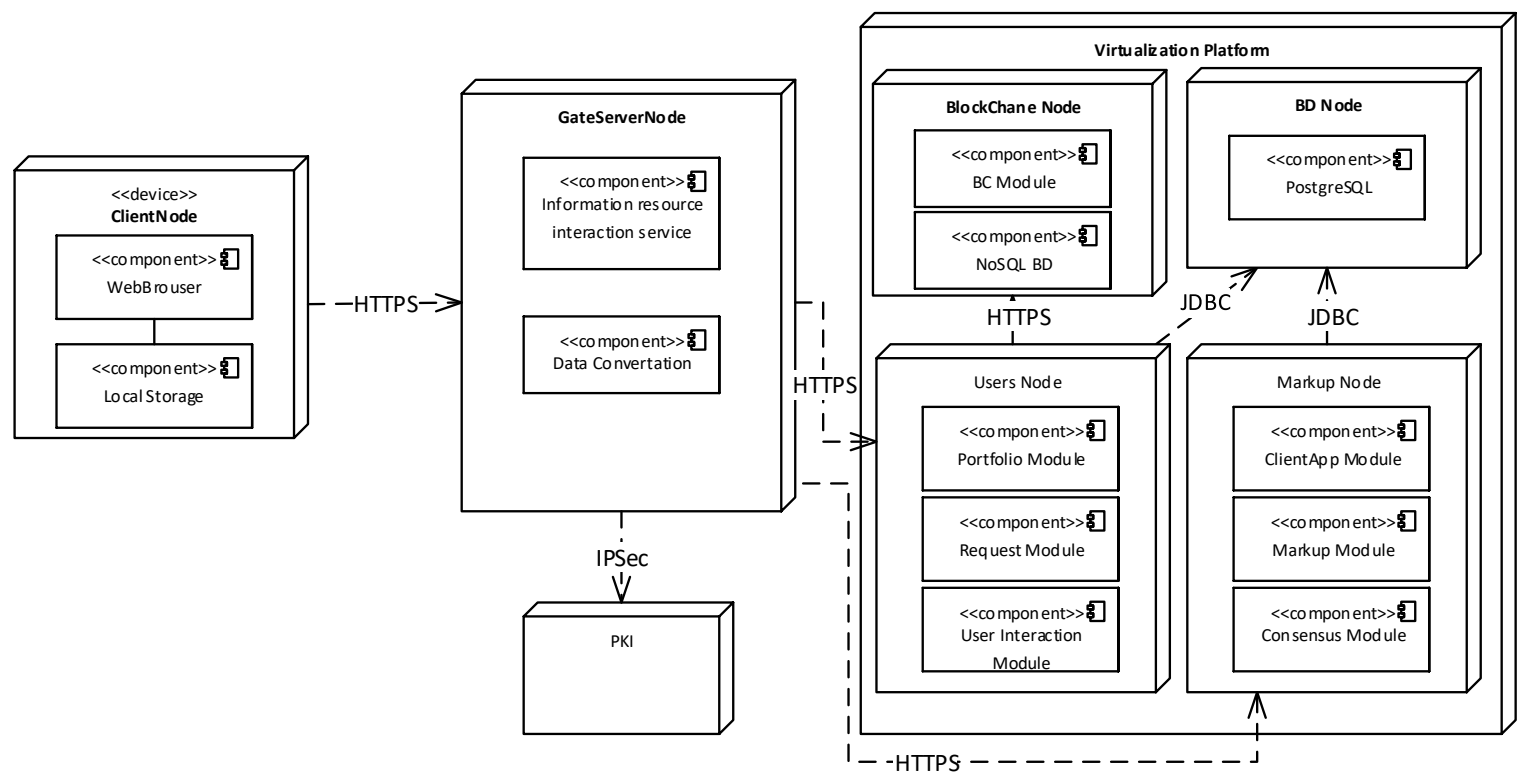

Figure 2. System architecture of the resource

The Users Node node contains modules for the work of patients (Request Module), a module for the work of physicians (Portfolio Module), and a module for user communication within the resource. The Markup Module node contains components for marking up images and comparative processing of reports to compile an expert rating. The rating calculation is based on direct and indirect parameters. The former includes the degree of coincidence of each markup with the markups of other experts, the latter - data on publications, academic degrees, work experience.

In order to fulfill the requirements for security, integrity, non-repudiation, accessibility (privacy, data integrity, signature, non-repudiation), blockchain technology elements are used to organize the storage of requests and reports. A private blockchain with a single node has been implemented with a full copy of the blockchain or full node (see BlockChain Node in Figure 2). This eliminated the fork problem in distributed storage. Both requests and responses are stored in a similar way. The first link in the chain is the request sent by the patient; if the expert takes the request into work, the next block is formed. If an expert decided to edit his report, a new block is formed in the same chain. For another expert, the reporting chain will be different, but with one start - initial request. A time stamp is added to each block, and the author's signature and author rating in the system existing at the time the report was created.

To implement the signing mechanism, a certificate is created for each user on an external PKI. The certificate is stored in a keychain protected by BlockChain Node. For patients, export of certificates is not provided. In the future, it is planned to implement certificate export or use of a user's personal certificate for medical experts.

The system architecture does not allow all actors except the Portal Owner to intervene in the blockchain (he can theoretically modify the blockchain due to the storage of certificates inside the resource), but even a modification by the Portal Owner can be detected due to time stamps. The blockchain stores data that cannot be deleted by users by end user agreement. Other data, such as user attributes in the portfolio, can be cleared. To store the rest of the data, a relational DBMS is used. 


\section{RESOURCE TESTING}

Currently, the developed resource is being prepared for launch on the platform of the ITMO University (St. Petersburg, Russia).

To this end, we conducted various types of resource testing. Among others, user testing was conducted using focus groups composed of typical users: an expert doctor ( 3 persons), medical students ( 7 persons), patients $(10$ persons). After working with the resource, the focus group participants were offered questionnaires with qualitative criteria that met the project goals and the formed requirements. We used a five-point scale. Estimates obtained and averaged are presented in Table 2.

Table 2. User Test Results

\begin{tabular}{|l|l|l|l|l|}
\hline \multicolumn{2}{|l|}{ Criteria (wording shortened) } & \multicolumn{2}{l|}{ Focus groups } \\
\cline { 3 - 5 } \multicolumn{2}{|l|}{} & Experts & Students & Patients \\
\hline 1 & Convenience of resource navigation & 4,7 & 4,9 & 4,5 \\
\hline 2 & Clarity and uniqueness of linguistic support & 4,9 & 4,3 & 3,7 \\
\hline 3 & Convenience of posting a request (pictures, medical history) & 4,5 & - & 4,7 \\
\hline 4 & Convenient image layout and report preparation & 4,8 & 4,8 & - \\
\hline 5 & Usefulness of a resource for skills formation & 4,5 & 4,9 & - \\
\hline 6 & Ease of requesting of colleagues ratings & 4,8 & 4,8 & - \\
\hline 7 & Ease of selecting requests for processing & 4,7 & 4,8 & - \\
\hline 8 & Ease of finding an expert for patients / mentor for students & 4,1 & 4,4 & 4,2 \\
\hline 9 & $\begin{array}{l}\text { The feasibility of using the resource for the development of } \\
\text { professional qualities }\end{array}$ & 5 & 5 & - \\
\hline
\end{tabular}

As can be seen from table 2, the resource as a whole received quite high user ratings. The relatively low scores given by patients on question 2 are explained by the fact that the medical history interface uses purely medical terms. Since the resource architecture provides for the use of different interfaces for different actors, this defect will be fixed in the next version of the system.

In addition, we conducted a security audit of the developed resource. The audit revealed problems with the configuration of KeyRings module security and network access to the BlockChain node. Identified deficiencies have been resolved.

\section{CONCLUSION}

The article addressed the issue of creation of a publicly accessible resource for increasing the volume of freely distributed medical datasets. The tasks set by the author of the article have been successfully fulfilled.

We identified the applications requirements that meet the main aspects for Internet resource of such kind, namely: community aspect (physical and virtual); labour market aspect (outsourcing, integration, mobility); security aspect (trust - security - privacy).

We developed and implemented the resource. The main business process of the resource and its system architecture are presented in the article.

We conducted experimental studies of the main functionalities of the developed service in terms of meeting the user's requirements as well as a security audit. Quite high estimates were obtained, which allows us to proceed to launch the resource on the platform of the ITMO University (St. Petersburg, Russia).

\section{ACKNOWLEDGEMENTS}

This work was financially supported by Russian Science Foundation, Grant 19-19-00696. 


\section{REFERENCES}

Allyn J. International Radiology Societies Tackle Radiologist Shortage. Feb. 20, 2020. https://www.rsna.org/en/news/2020/February/International-Radiology-Societies-And-Shortage

Boitsov V. et al. Software Tools for Manual Segmentation of Tomography Images Supporting Radiologist's Personal Context // Proceedings of the 25th Conference of Open Innovations Association FRUCT - 2019, pp. 64-76

Clinical radiology UK workforce census report 2018. https://www.rcr.ac.uk/publication/clinical-radiology-uk-workforcecensus-report-2018

Federal Law of November 21, 2011 No 323-Ф3 (as amended on 04.24.2020). http://www.pravo.gov.ru - 24.04.2020.

Fornell D. A Glimpse into Radiology in the Developing World in Africa. Dec. 08, 2017. https://www.itnonline.com/content/blogs/dave-fornell-itn-editor-rsna/glimpse-radiology-developing-world-africa

Goldman D.et al. Differential Motivations for Pursuing Interventional Radiology: Implications for Residency Recruitment. J Am Coll Radiol . 2019 Jan;16(1):82-88. doi: 10.1016/j.jacr.2018.08.002.

Han C. et al. Synthesizing diverse lung nodules wherever massively: 3D multi-conditional GAN-based CT image augmentation for object detection //2019 International Conference on 3D Vision (3DV). - IEEE, 2019. - C. 729-737.

Horos: Horos Project - Free DICOM Medical Image Viewer https://horosproject.org/

Improving radiology readiness in low-resource countries and regions. September 10, 2019. https://www.carestream.com/blog/2019/09/10/improving-radiology-readiness-in-low-resource-countries-and-regions/

Jin D. et al. CT-realistic lung nodule simulation from 3D conditional generative adversarial networks for robust lung segmentation //International Conference on Medical Image Computing and Computer-Assisted Intervention. - Springer, Cham, 2018. - C. 732-740.

Kumar S. N.et al. A voyage on medical image segmentation algorithms. Biomedical Research 2018; Special Issue: S75-S87

Lahat D., Adalı T., Jutten C. Multimodal Data Fusion: An Overview of Methods, Challenges and Prospects. Proceedings of the IEEE, Vol. 103, Is. 9, 2015

Mehrtash A., Pesteie M., Hetherington J., Behringer P.A., Kapur T., Wells W.M. III, Rohlinga R., Fedorov A., Abolmaesumi P. DeepInfer: Open-Source Deep Learning Deployment Toolkit for Image-Guided Therapy. Proc SPIE Int Soc Opt Eng. 2017 February 11; 10135: . doi:10.1117/12.2256011.

Norouzi A. et al. (2014) Medical Image Segmentation Methods, Algorithms, and Applications, IETE Technical Review, 31:3, 199-213, DOI: 10.1080/02564602.2014.906861

Orenstein B. W. Virtually Indispensable. Radiology Today. Vol. 19 No. 12 P. 18

Pool KJ.L. et al. A Structured Global Health Training Program for Radiology Residents. Journal of the American College of Radiology. February 2018 Volume 15, Issue 2, Pages 334-339

Rajalingam B., Priya R. Multimodality Medical Image Fusion Based on Hybrid Fusion Techniques. International Journal of Engineering and Manufacturing Science. Vol. 7, No. 1 (2017)

Ronneberger O., Fischer P., and Brox T. U-Net: Convolutional Networks for Biomedical Image Segmentation. arXiv:1505.04597v1 [cs.CV] 18 May 2015.

Ruggerio S., Weisser G. Integrating Mac Systems into a Medical IT Infrastructure: Creating an affordable radiology workstation with OsiriX. https://www.osirix-viewer.com/pdf/IntegratingMacSystems.pdf

Taylor C. S. et al. Generating Medical Student Interest in the Field of Radiology. Journal of the American College of Radiology. Journal of the American College of Radiology. February 2018 Volume 15, Issue 2, Pages 340-342

Vazirani A. A., O’Donoghue O., Brindley D. et al. Blockchain vehicles for efficient Medical Record management. npj Digit. Med. 3, 1 (2020). https://doi.org/10.1038/s41746-019-0211-0

Yen A.J. et al. The Stability of Factors Influencing the Choice of Medical Specialty Among Medical Students and Postgraduate Radiology Trainees. J Am Coll Radiol 2018;15: 886-891.

Sunshine J. H., and Meghea C. How Could the Radiologist Shortage Have Eased? American Journal of Roentgenology. Vol.187, Issue 5. 1160-1165. 10.2214/AJR.06.0559 